This item was submitted to Loughborough's Research Repository by the author.

Items in Figshare are protected by copyright, with all rights reserved, unless otherwise indicated.

\title{
Anxiety, anticipation and contextual information: a test of attentional control theory
}

PLEASE CITE THE PUBLISHED VERSION

http://dx.doi.org/10.1080/02699931.2015.1044424

\section{PUBLISHER}

(C) Taylor and Francis

\section{VERSION}

AM (Accepted Manuscript)

\section{PUBLISHER STATEMENT}

This work is made available according to the conditions of the Creative Commons Attribution-NonCommercialNoDerivatives 4.0 International (CC BY-NC-ND 4.0) licence. Full details of this licence are available at: https://creativecommons.org/licenses/by-nc-nd/4.0/

\section{LICENCE}

CC BY-NC-ND 4.0

\section{REPOSITORY RECORD}

Cocks, Adam J., Robin Jackson, Daniel T. Bishop, and A. Mark Williams. 2015. "Anxiety, Anticipation and Contextual Information: A Test of Attentional Control Theory”. Loughborough University.

https://hdl.handle.net/2134/20882. 
Anxiety, Anticipation, and Contextual Information: A Test of Attentional Control Theory

Adam J. Cocks, Robin C. Jackson, Daniel T. Bishop and A. Mark Williams

Department of Life Sciences, Brunel University London, Uxbridge, United Kingdom

Author Note

Adam J. Cocks, Robin C. Jackson, Daniel T. Bishop, and A. Mark Williams, Department of Life Sciences, Brunel University London, Uxbridge, United Kingdom.

The authors can state no conflicts of interest that would influence the outcome of the research.

Correspondence regarding this article should be addressed to Adam J. Cocks, Department of Life Sciences, College of Health and Life Sciences, Brunel University London, Kingston Lane, Uxbridge, Middlesex, UB8 3PH, United Kingdom. Email: Adam.Cocks@brunel.ac.uk 


\begin{abstract}
We tested the assumptions of Attentional Control Theory by examining the impact of anxiety on anticipation using a dynamic, time-constrained task. Moreover, we examined the involvement of high- and low-level cognitive processes in anticipation and how their importance may interact with anxiety. Skilled and less-skilled tennis players anticipated the shots of opponents under low- and high-anxiety conditions. Participants viewed three types of video stimuli, each depicting different levels of contextual information. Performance effectiveness (response accuracy) and processing efficiency (response accuracy divided by corresponding mental effort) were measured. Skilled players recorded higher levels of response accuracy and processing efficiency compared to less-skilled counterparts. Processing efficiency significantly decreased under high- compared to low-anxiety conditions. No difference in response accuracy was observed. When reviewing directional errors, anxiety was most detrimental to performance in the condition conveying only contextual information, suggesting that anxiety may have a greater impact on high-level (topdown) cognitive processes, potentially due to a shift in attentional control. Our findings provide partial support for Attentional Control Theory; anxiety elicited greater decrements in processing efficiency than performance effectiveness, possibly due to predominance of the stimulus-driven attentional system.
\end{abstract}

Keywords: skilled; tennis; perceptual-cognitive; processing efficiency; performance effectiveness. 
Anxiety, Anticipation, and Contextual Information: An Application of Attentional Control Theory

The impact of anxiety on performance has been a longstanding topic of debate in psychology. Several scientists have proposed theories to explain how anxiety affects performance (e.g., Cusp Catastrophe Model, Hardy, 1996, Hardy \& Parfitt, 1991; Processing Efficiency Theory [PET], Eysenck \& Calvo, 1992; Attentional Control Theory [ACT], Eysenck, Derakshan, Santos, \& Calvo, 2007). However, despite the wealth of research exploring the links between anxiety and performance, few researchers have assessed the impact of anxiety on anticipation judgements in time-constrained environments (for exceptions, see Smeeton, Williams, Hodges, \& Ward, 2005; Vickers \& Lewinski, 2012; Williams \& Elliott, 1999). Yet, the ability to process information and to anticipate the actions of others under severe time pressure is an essential component of performance in numerous domains such as sport, law enforcement, aviation, and military combat (Vickers \& Lewinski, 2012).

In the current paper, we test theoretical predictions arising from ACT (Eysenck et al., 2007) by examining the impact of anxiety on anticipation in tennis. In particular, we examine how anxiety influences the use of low- and high-level cognitive processes using the conceptual framework provided by ACT. We also investigate how anxiety influences the ability of skilled and less-skilled performers to anticipate an opponent’s actions under conditions where varying amounts of contextual information are presented. It has been reported that skilled performers are able to use contextual information, such as situational probabilities and tactical information, to facilitate anticipation judgements in timeconstrained domains (Abernethy, Gill, Parks, \& Packer, 2001; Crognier \& Féry 2005). 
Eysenck and colleagues (2007) proposed ACT as an expansion of Eysenck and Calvo’s (1992) Processing Efficiency Theory, which was developed to explain the relationship between anxiety and performance. Two main assumptions are outlined in the theory. First, that cognitive anxiety manifests itself in the form of worrisome thoughts; these thoughts impact working memory by depleting limited attentional resources, thereby reducing the amount of free attentional capacity to engage in coincident task demands. The second assumption is that anxiety increases motivation to avoid its negative effects, which leads to increased cognitive effort and the recruitment of additional processing resources (Eysenck \& Calvo, 1992). These two assumptions help to form the primary prediction of PET; that processing efficiency, which is an index of the individual resources invested to achieve a given level of performance, is negatively impacted more so than performance effectiveness, which is the level of performance indicated by a behavioural measure of a specified task (Eysenck, Payne, \& Derakshan, 2005). Specifically, because anxiety draws on one's attentional resources, processing efficiency suffers more greatly than does performance effectiveness.

ACT was proposed to address some of the limitations of PET (Eysenck et al., 2007). The theory predicts that high-anxiety will lead to allocation of attentional resources toward threat-related stimuli, be they external or internal (e.g., worrisome thoughts; Eysenck et al., 2007). This impairment and pre-empting of attentional resources leads to a shift in the attentional systems such that anxiety leads to increased reliance on the bottom-up, stimulusdriven attentional system, as opposed to the more top-down, goal-directed attentional system proposed by Corbetta and Schulman (2002) (Eysenck et al., 2007). In addition, ACT retains the main prediction of PET, namely that processing efficiency is impacted more so than performance effectiveness under anxiety-provoking conditions (Eysenck et al., 2007). 
Competitive sport affords a dynamic environment in which to test the applications of PET and ACT, as rapid decisions are required, with concomitantly high demands on perception, cognition, and action. It has been shown that expert sport performers are more adept at picking up low-level biological motion information from an opponent during anticipation (for reviews, see Müller \& Abernethy, 2012; Williams \& Ward, 2007). However, it has been reported that experts are able to use higher-order cognition to harness contextual information from a scene in order to inform their anticipation judgements (McRobert, Ward, Eccles, \& Williams, 2011). Rather than relying solely on kinematic cues, they formulate their decisions through the pickup of additional contextual information (e.g., situational probabilities; Abernethy et al., 2001). McRobert et al. (2011) reported that, when displaying video clips of a cricket bowler to skilled and less-skilled batters, both groups performed better when six bowls from the same bowler were displayed consecutively (high-context) in comparison to six deliveries from six different bowlers (low-context). This conceptualisation of the uses of contextual information is akin to the interactive encoding model proposed by Dittrich (1999). The model argues that the perception of biological motion within a visual scene involves the low-level, bottom-up structuring of motion information in combination with high-level, top-down encoding.

The impact of contextual information on anticipation has been shown in tennis. For example, expert performers within fast ball sports are able to decipher important cues from the probabilistic information available before the onset of any postural movements (Müller \& Abernethy, 2012). In addition, Farrow and Reid (2012) found that older, more highly-skilled tennis performers were more able to utilise game score information to produce faster responses to serves within a simulated match situation than were younger, less-skilled players. This latter finding suggests that situational probabilities associated with the specific game scenario play an important role within the initiation of anticipatory responses. Crognier 
and Féry (2005) reported that, when performing an in situ task, the ability of tennis players to employ tactical initiative and to harness situational probabilities determined whether they would successfully anticipate an opponent's attempted passing shots. The authors suggested that contextual information is generally masked within laboratory-based tests of anticipation, due to the relatively short duration of the video clips used. Moreover, the inclusion of court positioning information within a tennis scene benefits anticipation in skilled performers (Loffing, Wilkes \& Hagemann, 2011; Loffing \& Hagemann, 2014). However, it has yet to be ascertained whether the presence or absence of preceding shot sequences, along with the presence or absence of opponent postural information, has an impact upon the accuracy of anticipation judgements.

Since the use of contextual information implies the involvement of higher-order cognitive processes, this shift from low- to high-level cognitive processes should indicate increasing recruitment of the top-down, goal-directed attentional system housed within the dorsal frontoparietal network (Corbetta \& Schulman, 2002; Corbetta, Patel, \& Schulman, 2008). This system is guided by “current goals and pre-existing information about likely contingencies” (Corbetta et al., 2008, p.307) and is led by prior experience and representations of similar scenarios. In contrast, it may be suggested that the ability to pick up low-level biological motion is directed by the bottom-up, stimulus-driven attentional system, which “detects salient and behaviourally relevant stimuli in the environment” (Corbetta et al., 2008, p.306). Accordingly, the addition of contextual information to a scene should increase the contribution of the goal-directed attentional system relative to stimulus-driven, bottom-up processing of postural cues.

If the above assumption is to be carried forward, it is reasonable to propose that a shift from the goal-directed to the stimulus-driven attentional system should occur when under conditions of high-anxiety, as previously described within ACT (Eysenck et al., 2007), 
possibly leading to prioritisation of different sources of information. For example, it may be proposed that the impact of contextual information (e.g., the position of the players relative to the court, tennis ball, and each other) will be attenuated when a tennis player attempts to predict the outcome of an opponent's actions under anxiogenic conditions, as the stimulusdriven processing of postural cues, for example predominates.

In the current study, we test some of the predictions of ACT using a tennis anticipation paradigm. Specifically, we examine how state anxiety influences the use of lowand high-level cognitive processes, with regards to the prioritisation of different informational sources when varying levels of contextual information are available. In this case, the level of contextual information is varied by manipulating the availability of postural, shot sequencing, and court position information. The impacts of these manipulations are observed within skilled and less-skilled tennis players. We propose a number of hypotheses. First, it is predicted that overall processing efficiency will be lower under anxiety-provoking conditions, in the absence of increased performance effectiveness (response accuracy).

Second, although the manipulation of contextual information coupled with anxiety induction is somewhat exploratory, we hypothesise that reduced performance and processing efficiency, along with an increased number of directional and depth errors will occur within trials that make no postural cue information available in comparison to the other conditions. The latter differentiation is especially expected under high-anxiety as the stimulus-driven system will have little salient information to which it can attend. Finally, in accordance with the expertise literature, we hypothesise that skilled performers will produce greater response accuracy and fewer directional and depth errors than low-skilled performers.

\section{Method}

\section{Participants}


A total of 12 skilled (mean age $=20.67$ years, $S D=2.35$ yrs) and 12 less-skilled (mean age $=21.83$ years, $S D=3.51 \mathrm{yrs}$ ) male tennis players participated. This sample size is larger than previously employed by researchers who have reported significant effects when testing the uses of contextual information (see McRobert et al., 2011) and the impact of anxiety on anticipation (see Williams \& Elliott, 1999). The skilled participants had British Tennis Membership Ratings (BTMR) between 10.2 and 8.1 and a mean of 10.54 years (SD = 4.41 yrs) tennis playing experience. The less-skilled group did not possess a BTMR and had a mean of 7.75 years $(S D=7.31$ yrs) tennis playing experience. Written and informed consent was obtained from all participants and Institutional Research Ethics Committee Approval was obtained from the lead institution.

\section{Test Stimuli}

Three types of video sequences were created for the experiment. The first showed only the opponent's postural information; that is, all court markings and previous shot sequences were removed from the original video sequence (Fig. 1a). The second was a computer animation developed using Hawk-Eye (Hawk-Eye Innovations Ltd., Basingstoke, UK) data that depicted the dynamic court position of both players represented by cylinders, as well as court markings and the ball position (Fig. 1b). The final clip type showed the original video sequence containing the players, ball, and court markings (Fig. 1c).

\section{$<$ INSERT FIGURE 1 HERE $>$}

Figure 1a was filmed with a high definition video camera (Sony Handycam HDRPJ420VE, Tokyo, Japan) mounted on a wall which was 1.90 metres above ground level and 6.40 metres behind the baseline at the end of the court. This camera was trained on the player at the far end of the court and zoomed in by 2x optical zoom so as to attempt to remove the player on the near side of the court from view. The footage obtained was then post-produced 
using Pinnacle Studio 15 (California, USA) to remove all court markings from view leaving visible just the 'opposing' player (the player at the end of the court opposite to the video camera), part of the net, and some of the background immediately behind the opposing player. Figure 1c was filmed with a high definition wide-angle lens video camera (Contour ROAM, Seattle, USA) from the same positional dimensions as the previously mentioned camera, enabling all aspects of the court and surrounding areas to be present within this stimulus condition. The animations of the video sequences (see Fig. 1b) were developed using 3-D coordinates of both players and the ball trajectory captured using the Hawk-Eye system. These coordinates were then fed into the Lawn Tennis Association (LTA) Rendering Engine (Julien Pansiot, London, UK) that produced the finished animations, which retained the court markings and ball movement and depicted the players as vertical cylinders.

The stimuli chosen for the experiment were selected using criteria delineated by LTA coaches, which depicted the most likely situations whereby anticipation would be necessary within tennis (e.g., when the opponent would be performing more attacking shots, such as passing shots and drop shots). A total of 48 match situations (12 for each directional outcome; short left, short right, deep left and deep right) were chosen to be displayed in the three contextual conditions, generating a total of 144 test stimuli. The postural cue only condition (Fig. 1a) comprised a single shot executed by the on-screen performer occluded at ball-racket contact, whereas the animation (Fig. 1b) and the wide-angle (Fig. 1c) clips began from the serve through to occlusion of the target shot at racket-ball contact. The 144 test stimuli were divided into 12 blocks of 12 trials. The inter-trial interval was set at 4 seconds, with the inter-block interval set at 15 seconds at which time the instructions of the task were re-presented. Additionally, only a single form of video stimuli was presented in each block, so as to avoid possible annoyance due to differences in displayed stimuli (see Loffing et al., 2011). 


\section{Apparatus}

Film clips were projected using an Optoma HD25 2-D projector (Watford, UK) onto the wall of the testing area, creating a $1.68 \mathrm{~m} \times 2.20 \mathrm{~m}$ image size. Participants stood 3.92 metres away from the screen, such that the player, when stood on the far baseline, subtended a vertical visual angle of 2.56 degrees and 3.14 degrees when stood at the net. The four rectangular segments, representing the four areas of the 'near side' court in which the ball could land, were marked out at the participants' feet, with each rectangle measuring $46 \mathrm{~cm} \mathrm{x}$ $80 \mathrm{~cm}$ in size (see Fig. 2 for schematic).

\section{Design and Measures}

The experiment employed a 2 (Skill Level) $\times 2$ (Anxiety Condition) $\times 3$ (Context Condition) mixed-factor design; Anxiety and Context Conditions were within-participants factors. Response accuracy and processing efficiency (response accuracy divided by mental effort ratings) were the dependent variables.

State Anxiety. Competitive state anxiety was measured using the Mental Readiness Form-3 (MRF-3; Krane, 1994). This 3-item questionnaire was completed by participants after each block of 12 trials. Each item required a response on an 11-point Likert scale from 'not worried' (1) to 'worried' (11) for cognitive anxiety; 'not tense' (1) to 'tense' (11) for somatic anxiety; and 'not confident' (1) and 'confident' (11) for self-confidence. The MRF-3 was incorporated to keep in line with previous research into the application of PET and ACT to sport (see Wilson, Wood, \& Vine, 2009; Wood \& Wilson, 2011).

Response Accuracy. After each video was occluded, participants were asked to indicate where on the court they thought the ball would land by stepping onto one of four rectangular zones displayed in a $2 \times 2$ numbered grid on the floor of the testing area $(1=$ 
short left, 2 = short right, $3=$ deep left and $4=$ deep right) and to state the associated grid number (see Fig. 2). Response accuracy was measured as the percentage of correct responses produced by the participants.

Processing Efficiency. The Rating Scale for Mental Effort (RSME; Zijlstra, 1993) is a uni-dimensional scale for measuring cognitive effort that has been validated in an array of experimental settings (see Zijlstra, 1993). The scale requires the respondent to make a mark on a vertical line comprising ratings from 0 to 150 to denote their perceived mental effort on a task at that point in time. Nine descriptors are used as anchors, such that a score of 2 relates to "absolutely no effort”, 58 to "rather much effort” and 113 to "extreme effort” (Veltman \& Gaillard, 1996). This subjective means of rating mental effort has been "used as an operationalization of processing efficiency” (Zijlstra, 1993, p.137). A processing efficiency quotient was calculated by dividing participants' response accuracy score by the associated RSME rating. The scale was also used to assess cognitive demands between the different stimulus presentation conditions.

\section{Procedure and Experimental Conditions}

Prior to commencing the task, participants were made aware of the test procedure and were asked to complete an informed consent sheet and demographic questionnaire.

Participants were presented with 15 practice trials, consisting of 5 trials within each of the 3 conditions to enable familiarisation with the task. Following each clip, they were asked to step in the relevant segment and state the number of the segment in which they thought the ball would bounce after the occlusion of the clips. Once the practice phase was completed, the experimental trials began and the participants completed 3 blocks of 12 trials under lowanxiety, followed by 6 blocks of 12 trials under anxiogenic conditions and then finally a 
second low-anxiety phase consisting of 3 blocks of 12 trials. Participants' responses were recorded via a laptop using spreadsheet software.

All participants performed the task under high and low-anxiety conditions, in an A-BA (low-anxiety, high-anxiety, low-anxiety) design. In the first low-anxiety condition, participants were asked to imagine they were in a match environment and were told that their task was to try to judge the direction of the final shot in the sequence. There was no feedback given to the participants when under low-anxiety conditions.

In the high-anxiety condition, participants were told that their performance was to be evaluated by a certified LTA coach, in order to elicit feelings of evaluation apprehension. In order to further induce anxiety, a video camera (Sony Handycam HDR-PJ420VE, Tokyo, Japan) was placed behind the participant so as to film both the participant and the screen (for an example, see Beilock \& Carr, 2005; DeCaro, Thomas, Albert, \& Beilock, 2011; [see Fig. 2]). The participants were told that the camera was intended to help provide the LTA coach with feedback in order to see in which conditions the participants were struggling. In conjunction with attempting to provoke evaluation apprehension, participants were provided with false feedback that they were performing below what was expected of a player at their level. Participants were told that they needed to improve their performance by at least $10 \%$ in order to (i) score above average for their BTMR and (ii) be able to complete the task successfully; this goal was reinforced throughout the high-anxiety phase by giving false response accuracy feedback after every block of trials.

For the second set of low-anxiety trials, participants were informed that the evaluation period was over and the video camera was removed from the testing area. The participants were then given a break of approximately three minutes and asked to continue for the final three blocks and to keep on trying their best. The MRF-3 and RSME were completed by the 
participants following each block of trials. The order of the blocks was counterbalanced between anxiety conditions. Each session took no more than 60 minutes to complete.

\section{$<$ INSERT FIGURE 2 HERE $>$}

\section{Data Analysis}

Prior to conducting the main analyses, a preliminary analysis was undertaken to ensure that the separate low-anxiety conditions could be combined. Paired samples t-tests analysed response accuracy, processing efficiency, cognitive and somatic anxiety ratings between the respective conditions. All comparisons were found to be non-significant ( $p \geq$ .18), therefore the conditions were pooled.

The calculated quotient for processing efficiency and response accuracy scores were submitted to separate $2 \times 2 \times 3$ (Skill Level [skilled, less-skilled] $\times$ Anxiety [low, high] $\times$ Contextual Information [postural cue, animation, wide angle]) mixed-factor analysis of variance (ANOVA). In addition, two separate mixed-factor ANOVAs were run on directional and depth errors when responding to the ball bounce location to investigate how the participants' incorrect responses were manifested. Effect size is reported as partial eta squared $\left(\eta_{\mathrm{p}}{ }^{2}\right)$. Any significant effects were followed up by pairwise comparisons with Bonferroni adjustments. Significant interaction effects were subject to separate ANOVAs for each contextual condition, subsequent to this, independent samples and paired-samples t-tests were conducted on any resultant two-way interaction effects. Paired samples t-tests were run on MRF-3 scale ratings to check whether anxiety manipulations had been successful.

\section{Results}

Mental Readiness Form-3 (MRF-3) 
The t-tests revealed a significant effect of anxiety condition for all three variables, with participants reporting significantly higher cognitive anxiety ratings within the high- ( $M$ $=4.66, S E=0.44)$ in comparison to the low $-(M=3.50, S E=0.32)$ anxiety condition, $t(23)=$ 5.21, $p<.001$. Similarly, participants reported significantly higher somatic anxiety during the high- $(M=4.34, S E=0.41)$ when compared to the low-anxiety $(M=3.91, S E=0.34)$ condition, $t(23)=2.54, p<.05$, and were less confident in the high- $(M=5.40, S E=0.29)$ compared with the low- $(M=6.13, S E=0.26)$ anxiety condition, $t(23)=5.61, p<.001$.

\section{Response Accuracy and Processing Efficiency}

Response accuracy. There was a significant main effect of skill level, $F(1,22)=$ 23.72, $p<.001, \eta_{\mathrm{p}}{ }^{2}=0.52$. The skilled group $(M=53.82, S E=1.66 \%)$ made a higher proportion of correct responses than the less-skilled group $(M=42.42, S E=1.66 \%)$ A significant main effect of contextual condition was observed, $F(2,44)=74.33, p<.001, \eta_{\mathrm{p}}{ }^{2}$ $=0.77$, reflecting significantly higher response accuracy in the postural cue condition $(M=$ 59.29, $S E=2.04 \%)$ than in the animation $(M=36.98, S E=1.08 \%, p<.001)$ and wide angle $(M=48.09, S E=1.47 \%, p<.001)$ conditions. Moreover, significantly higher response accuracy was observed within the wide angle condition in comparison to the animation condition $(p<.001)$ (see Fig. 3). The main effect of anxiety condition was non-significant, $F$ $(1,22)=0.06, p>.05, \eta_{\mathrm{p}}{ }^{2}=0.00$, as were all two-way and higher-order interaction effects, $F \mathrm{~s}$ $\leq 1.45, p s \geq .25$.

\section{$<$ INSERT FIGURE 3 HERE $>$}

Processing efficiency. There was a significant main effect of skill level, $F(1,22)=$ 10.27, $p<.01, \eta_{\mathrm{p}}{ }^{2}=0.32$. Skilled players $(M=1.07, S E=0.10)$ were more efficient than their less-skilled counterparts $(M=0.63, S E=0.10)$. The anxiety condition also impaired processing efficiency, $F(1,22)=20.11, p<.001, \eta_{\mathrm{p}}{ }^{2}=0.48$. Reduced processing efficiency 
was reported in the high-anxiety $(M=0.92, S E=0.08)$ compared with the low-anxiety $(M=$ $0.78, S E=0.60)$ condition. Also, a main effect of contextual condition was observed, $F(2$, $44)=51.94, p<.001, \eta_{\mathrm{p}}^{2}=0.70$. Performance in the animation condition $(M=0.55, S E=$ $0.05)$ was significantly less efficient than that in both the postural cue $(M=1.14, S E=0.09, p$ $<.001)$ and wide angle $(M=0.85, S E=0.08, p<.001)$ conditions. There was also a significant difference between the latter two conditions, $(p<.001)$. All interaction effects were non-significant, $F \mathrm{~s} \leq 4.11, p s>.05$.

\section{Directional and Depth Errors}

Directional errors. ANOVA revealed a significant main effect of skill level, $F$ (1, $22)=15.52, p<.01, \eta_{\mathrm{p}}{ }^{2}=0.41$. The less-skilled performers $(M=19.97, S E=0.85 \%)$ made significantly more directional errors than the skilled performers $(M=15.22, S E=0.85 \%)$. A significant main effect of contextual condition was also observed, $F(2,44)=16.78, p<.001$, $\eta_{\mathrm{p}}{ }^{2}=0.43$. There were significantly more errors committed in the wide angle condition $(M=$ 22.40, $S E=0.85 \%)$ than in the postural cue $(M=13.37, S E=1.43 \%, p<.001)$ and animation $(M=17.01, S E=0.89 \%, p<.01)$ conditions; however, there was no difference between postural cue and animation conditions. A significant Anxiety Condition $\times$ Contextual Condition $\times$ Skill Level interaction was found, $F(2,44)=3.65, p<.05, \eta_{\mathrm{p}}{ }^{2}=0.14$. All other main and interaction effects were non-significant, $F s \leq 1.34$, $p s \geq .26$.

The three-way interaction was followed up using ANOVAs on the separate contextual conditions. In the animation condition a significant Anxiety $\times$ Skill Level interaction was found, $F(1,22)=5.11, p<.05, \eta_{\mathrm{p}}^{2}=0.19$. This interaction was caused by the low-skilled group making significantly more directional errors $(M=20.14, S E=1.76 \%)$ than the skilled group $(M=11.11, S E=1.57 \%)$ under low anxiety, $p<.01$, but not under high anxiety, $p=$ .83. In addition, the skilled group produced significantly more directional errors in the high- 
anxiety $(M=18.75, S E=2.02 \%)$ than low-anxiety $(M=11.11, S E=1.57 \%)$ condition, $t(11)$ $=2.88, p<.05$. No significant difference was found when comparing the performance of low-skilled players, $t(11)=0.62, p=.55$ (see Fig. 4). In the wide angle condition, a significant main effect of skill level was revealed, $F(1,22)=8.18, p<.01, \eta_{\mathrm{p}}{ }^{2}=0.27$, caused by low-skilled performers $(M=19.10, S E=1.25 \% ; M=24.83, S E=1.20 \%)$ making significantly more direction errors than skilled performers $(M=14.93, S E=1.25 \% ; M=$ 19.97, $S E=1.20 \%$ ). All other main and interaction effects were non-significant, $F \mathrm{~s} \leq 3.32$, $p \mathrm{~s}$ $\geq .08$.

\section{$<$ INSERT FIGURE 4 HERE $>$}

Depth errors. ANOVA revealed a significant main effect of contextual condition, $F$ $(2,44)=14.45, p<.001, \eta_{\mathrm{p}}{ }^{2}=0.40$. Significantly more depth errors were made when responding to the animation condition $(M=25.78, S E=1.44 \%)$ than both the postural cue ( $M$ $=18.84, S E=1.36 \%, p<.01)$ and wide angle $(M=18.14, S E=1.32 \%, p<.01)$ conditions, although, there was no significant difference between the postural cue and wide angle conditions. All other main and interaction effects were non-significant, $F \mathrm{~s} \leq 2.04$, $p s \geq .17$.

\section{Discussion}

We used a novel method to test the predictions of ACT (Eysenck et al., 2007). Moreover, we examined whether anxiety impacted upon the use of contextual information when making anticipation judgements. Also, in line with the predictions of ACT we examined changes in processing efficiency and performance effectiveness under anxiogenic circumstances. In addition, we examined the effects of anxiety upon anticipation judgements amongst skilled and less-skilled tennis players. 
Our findings show that levels of anxiety were successfully manipulated, with participants reporting higher levels of cognitive and somatic anxiety and lower levels of selfconfidence when under anxiogenic circumstances compared to low-anxiety conditions. The change in cognitive anxiety was the primary focus, as anxiety is said to heighten the production of worrisome thoughts, which augment the demands on working memory (Eysenck \& Calvo, 1992). The experimental manipulations produced a sustained effect on anxiety levels, as scores were reported by the participants following every block of trials resulting in the production of a mean score. The different procedure employed may be the reason for the relatively small change in anxiety across blocks when compared with other published reports (e.g., Wilson et al., 2009).

In accordance with ACT (Eysenck et al., 2007), it was predicted that performance effectiveness would be maintained at the expense of processing efficiency on the task. Support was offered for this particular hypothesis as processing efficiency significantly decreased during the high-anxiety condition, whereas no change in response accuracy was observed. The decreases in processing efficiency under anxiety conditions supports the findings of previous researchers who have indicated an increase in cognitive investment, as measured by the RSME (Causer, Holmes, Smith, \& Williams, 2011; Smith, Bellamy, Collins, \& Newell, 2001). Eysenck and Calvo (1992) proposed that anxiety places additional demands on working memory, thereby requiring more cognitive resources to be recruited in order for the same performance levels to be maintained. Our data show that in order to maintain anticipatory performance under anxiety, cognitive engagement and effort needs to be increased (cf., Eysenck et al., 2007).

The exploratory prediction that anxiety would result in a reduced ability to harness contextual information (shot sequencing and players' court positioning) was partially supported. It was proposed that under high-anxiety conditions, the tendency of participants to 
use contextual information would be reduced and that this would be evidenced by a reduction in response accuracy when postural cues were not present, due to disengagement of the goaldirected attentional system (Eysenck et al., 2007). However, results for the overall response accuracy scores showed this not to be the case. A reason behind this finding could be offered by the motivational aspects of anxiety, clarified by Eysenck and Derakshan (2011) in relation to ACT. These authors suggested that high-anxiety during a demanding task with clear task goals can lead to overriding of the stimulus-driven attentional system due to anxiety increased motivation and the use of compensatory strategies, such as increased mental effort. These strategies may therefore have been sufficient to prevent the overall shift between the information sources used within the anticipation paradigm employed in this study.

The interpretation of the directional errors made by the participants offers partial support for our hypothesis. A three-way interaction was reported between skill level, anxiety, and contextual condition when analysing the scenarios where directional errors were made. It was revealed that lateral direction judgements in the skilled group were impaired in the highanxiety condition when responding to the animated video clips. It appears that the ability of skilled players to harness top-down contextual information when judging laterally was negatively affected under high-anxiety conditions. According to ACT, this effect could be accounted for by the predominance of the stimulus-driven attentional system under anxietyprovoking conditions (Eysenck et al., 2007) coupled with the lack of salient, low-level biological motion information in this condition to which it could orientate attention. Therefore, as skilled performers are known to be better able to utilise contextual information (Farrow \& Reid, 2012; Müller \& Abernethy, 2012) and the presence of anxiety reduces their ability to incorporate this advantage, their ability to produce lateral judgements is reduced.

In addition to the fact that the use of contextual information was moderately impacted by increases in anxiety levels, there were still differences in both processing efficiency and 
response accuracy when comparing the conditions presenting differing levels of contextual information. Decreased processing efficiency was evident when postural cues were not present within the stimulus display, therefore suggesting that the ability to infer anticipatory responses without access to kinematic information is a more mentally demanding task. The animation condition also yielded the lowest proportion of correct responses. However, it should be noted that response accuracy within the animation condition is well above the level of chance, indicating that the presence of contextual information alone provides sufficient information to facilitate anticipation. The combination of these particular results implies that the presence of postural cue information is paramount in producing autonomous and accurate anticipation judgements (Loffing et al., 2011).

Along with errors in direction, the ability to anticipate the depth of the shots was impacted by the type of presented stimuli. Significantly more depth errors were made when viewing the animation condition than the other two types of stimulus presentation. A possible reason for this result is that the absence of postural information makes it difficult to perceive the angle of the racket, therefore not providing the performers with ample information to make an informed decision (Cañal-Bruland, van Ginneken, van der Meer, \& Williams, 2011).

Our findings show that there was a significant difference between the skilled and lessskilled tennis performers in response accuracy. This is an extremely robust finding within expertise research, as higher skill levels constantly give rise to superior anticipatory responses and thereby performance (Mann, Williams, Ward, \& Janelle, 2007). Skilled performers possess greater perceptual-cognitive expertise when compared to their less-skilled counterparts, leading to a greater ability in making use of postural cue and situation specific probabilistic information (Müller \& Abernethy, 2012). 
With regards to future research, the specific attentional mechanisms involved in the regulation of stimulus type prioritisation during anticipation under anxiety should be considered. While the study highlights the impact of anxiety on the use of higher-order cognition, we can only speculate on the precise nature of the attentional mechanisms involved. Moreover, the relative interaction between participant skill level and the use of contextual information under anxiety merits further investigation. Additionally, further empirical work is needed to examine the applicability of ACT to other dynamic, timeconstrained environments, not just confined to sport in order to provide a broader test of the theory’s applicability and generalisability.

In summary, we explored the impact of anxiety on anticipation and tested the postulations of ACT. Our findings provide partial support for the ACT theoretical framework, because processing efficiency was more significantly affected than performance effectiveness when tennis players made anticipatory judgments under high-anxiety conditions. Moreover, anxiety was shown to impact the ability of skilled performers to use contextual information, suggesting that anxiety may have impacted more greatly on the processing of high-level, rather than low-level, cognitive processes. In line with the propositions of ACT, the reduced ability to use such top-down information may be due to a prioritisation of the stimulus-driven attentional system. Overall, the current study provides support for Attentional Control Theory using a novel and dynamic task. The results clarify how anxiety impacts on different perceptual-cognitive processes underpinning anticipation and broadens the scope and applicability of ACT, although further work would help to clarify the attentional mechanisms involved in the observed effects. 


\section{References}

Abernethy, B., Gill, D. P., Parks, S. L., \& Packer, S. T. (2001). Expertise and the perception of kinematic and situational probability information. Perception, 30, 233-252. doi: 10.1068/p2872

Beilock, S. L., \& Carr, T. H. (2005). When high-powered people fail: Working memory and "chocking under pressure" in math. Psychological Science, 16, 101-105.

Cañal-Bruland, R., van Ginneken, W. F., van der Meer, B. R., \& Williams, A. M. (2011). The effect of local kinematic changes on anticipation judgements. Human Movement Science, 30, 495-503. doi: 10.1016/j.humov.2010.10.001

Causer, J., Holmes, P. S., Smith, N. C., \& Williams, A. M. (2011). Anxiety, movement kinematics, and visual attention in elite-level performers. Emotion, 11, 595-602. doi: $10.1037 / \mathrm{a} 0023225$

Corbetta, M., \& Schulman, G. L. (2002). Control of goal-directed and stimulus-driven attention in the brain. Nature Reviews Neuroscience, 26, 105-125. doi: $10.1038 / \mathrm{nrn} 755$

Corbetta, M., Patel, G., \& Schulman, G. L. (2008). The reorienting system of the human brain: From environment to theory of mind. Neuron, 58, 306-324. doi: 10.1016/j.neuron.2008.04.017

Crognier, L., \& Fréy, Y.-A. (2005). Effect of tactical initiative on predicting passing shots in tennis. Applied Cognitive Psychology, 19, 637-649. doi: 10.1002/acp.1100 
DeCaro, M. S., Thomas, R. D., Albert, N. B., \& Beilock, S. L. (2011). Chocking under pressure: Multiple routes to skill failure. Journal of Experimental Psychology: General, 140, 390-406. doi: 10.1037/a0023466

Dittrich, W. H. (1999). Seeing biological motion: Is there a role for cognitive strategies? In A. Braffort, R. Gherbi, S. Gibet, J. Richardson, \& D. Teil (Eds.), Gesture-based communication in human-computer interaction (pp. 3-22). Berlin: Springer-Verlag.

Eysenck, M. W., \& Calvo, M. G. (1992). Anxiety and performance: The processing efficiency theory. Cognition and Emotion, 6, 409-434. doi: $10.1080 / 02699939208409696$

Eysenck, M. W., \& Derakshan, N. (2011). New perspectives in attentional control theory. Personality and Individual Differences, 50, 955-960. doi: 10.1016/j.paid.2010.08.019

Eysenck, M. W., Derakshan, N., Santos, R., \& Calvo, M. G. (2007). Anxiety and cognitive performance: Attentional control theory. Emotion, 7, 336-353. doi: 10.1037/15283542.7.2.336

Eysenck, M. W., Payne, S., \& Derakshan, N. (2005). Trait anxiety, visuospatial processing, and working memory. Cognition and Emotion, 19, 1214-1228. doi: $10.1080 / 02699930500260245$

Farrow, D., \& Reid, M. (2012). The contribution of situational probability information to anticipatory skill. Journal of Science and Medicine in Sport, 15, 368-373. doi: 10.1016/j.jsams.2011.12.007

Hardy, L. (1996). Testing the predictions of the cusp catastrophe model of anxiety and performance. The Sport Psychologist, 10, 140-156. 
Hardy, L., \& Parfitt, G. (1991). A catastrophe model of anxiety and performance. British Journal of Psychology, 82, 163-178.

Krane, V. (1994). The mental readiness form as a measure of competitive state anxiety. The Sport Psychologist, 8, 189-202.

Loffing, F., \& Hagemann, N. (2014). On-court position influences skilled players' anticipation of shot outcome. Journal of Sport \& Exercise Psychology, 36, 14-26. doi: 10.1123/jsep.2013-0082

Loffing, F., Wilkes, T., \& Hagemann, N. (2011). Skill level and graphical detail chap perceptual judgements in tennis. Perception, 40, 1447-1456. doi: 10.1068/p7035

Mann, D. T. Y., Williams, A. M., Ward, P., \& Janelle, C. M. (2007). Perceptual-cognitive expertise in sport: A meta-analysis. Journal of Sport and Exercise Psychology, 29, 457-478.

McRobert, A. P., Ward, P., Eccles, D. W., \& Williams, A. M. (2011). The effect of manipulating context-specific information on perceptual-cognitive processes during a simulated anticipation task. British Journal of Psychology, 102, 519-534. doi: 10.1111/j.2044-8295.2010.02013.x

Müller, S., \& Abernethy, B. (2012). Expert anticipatory skill in striking sports: A review and a model. Research Quarterly for Exercise and Sport, 83, 175-187.

Smeeton, N. J., Williams, A. M., Hodges, N. J., \& Ward, P. (2005). The relative effectiveness of various instructional approaches in developing anticipation skill. Journal of Experimental Psychology: Applied, 11, 98-110. doi: 10.1037/1076-898X.11.2.98 
Smith, N. C., Bellamy, M., Collins, D. J., \& Newell, D. (2001). A test of processing efficiency theory in a team sport context. Journal of Sports Sciences, 19, 321-332.

Veltman, J. A., \& Gaillard, A. W. K. (1996). Physiological indices of workload in a simulated flight task. Biological Psychology, 42, 323-342.

Vickers, J. N. \& Lewinski, W. (2012). Performing under pressure: Gaze control, decision making and shooting performance of elite and rookie police officers. Human Movement Science, 31, 101-117. doi: 10.1016/j.humov.2011.04.004

Williams, A. M., \& Elliott, D. (1999). Anxiety, expertise, and visual search strategy in karate. Journal of Sport \& Exercise Psychology, 21, 362-375.

Williams, A. M., \& Ward, P. (2007). Perceptual-cognitive expertise in sport: Exploring new horizons. In G. Tenenbaum, \& R. C. Eklund (Eds.), Handbook of Sport Psychology (pp. 203-223). New York: John Wiley and Sons.

Wilson, M. R., Wood, G., \& Vine, S. J. (2009). Anxiety, attentional control, and performance impairments in penalty kicks. Journal of Sport \& Exercise Psychology, 31, 761-775.

Wood, G., \& Wilson, M. R. (2011). Quiet-eye training for soccer penalty kicks. Cognitive Processing, 12, 257-266. doi: 10.1007/s10339-011-0393-0

Zijlstra, F. R. H. (1993). Efficiency in working behaviour: A design approach for modern tools. Delft: Delft University Press. 


\section{Figure Captions}

Figure 1. Screenshots of the three types of experimental stimuli.

Figure 2. Schematic of the experimental set-up viewed from above.

Figure 3. Mean response accuracy (\%) and standard error of the three contextual information conditions.

Figure 4. Mean directional errors (\%) and standard error of the animation contextual information condition. 